\title{
Integrated Content and Format Checking for Processing of SCP ECG Records
}

\author{
C Zywietz $^{1}$, R Fischer ${ }^{2}$ \\ ${ }^{1}$ BIOSIGNA Institute, Hannover, Germany \\ ${ }^{2}$ Medical School Hannover, Hannover, Germany
}

\begin{abstract}
Interoperability between medical devices and between host systems is a key requirement for the establishment of an electronic patient health record. A pre-requisite for interoperability is standardisation of message formats and of messaging protocols. We report on the development of testing tools for compliance checks of the $S C P-E C G$ interchange format with the specifications of the CEN standard prENV1064. The presented tools are important for development as well as compliance testing of SCP formatted ECG records. They are necessary to promote the interoperability of important non-invasively gathered cardiac function information. This information is increasingly to be integrated into the electronic patient health record and to be interchanged between the various health care providers. Practical tests in our laboratory provided useful information for our own and external SCP implementations.
\end{abstract}

\section{Introduction}

Interoperability between medical devices and between host systems is a key requirement for the establishment of an electronic patient health record. A pre-requisite for interoperability is standardisation of message formats and of messaging protocols.

For computer assisted electrocardiography a specific standard: SCP-ECG has been developed in 1993 [1]. This standard specifies the interchange format and a messaging procedure for ECG cart-to-host communication and for retrieval of SCP-ECG records from the host (to the ECG cart).

The basis for the SCP Standard has been developed during a European AIM R\&D project 1989-1991. During this project an inventory on existing ECG compression methods has been made and a new approach for quality assured ECG signal compression has been developed [2].

In 1993 the "SCP Standard Communication Protocol" was approved by CEN as a pre-Standard ENV 1064 [3].

The aim of our work was to develop tools for verification of the compliance of SCP implementations with the specifications given by the SCP standard document ENV 1064. The SCP standard specifies that the information is to be structured in data sections as shown in the table 1.

Table 1. Structure of a SCP-ECG record

\begin{tabular}{|c|c|}
\hline Mandatory & $\begin{array}{c}2 \text { BYTES - CHECKSUM - CRC - CCITT OVER THE } \\
\text { ENTIRE RECORD } \\
\text { (EXCLUDING THIS WORD })\end{array}$ \\
\hline Mandatory & $\begin{array}{c}4 \text { BYTES - (UNSIGNED) SIZE OF THE ENTIRE ECG } \\
\text { RECORD (IN BYTES) }\end{array}$ \\
\hline Mandatory & $\begin{array}{l}\text { (Section 0) } \\
\text { POINTERS TO DATA AREAS IN THE RECORD }\end{array}$ \\
\hline Mandatory & $\begin{array}{c}\text { (Section 1) } \\
\text { HEADER INFORMATION - PATIENT DATA/ECG } \\
\text { ACQUISITION DATA }\end{array}$ \\
\hline Optional & $\begin{array}{c}\text { (Section 2) } \\
\text { HUFFMAN TABLES USED IN ENCODING OF ECG } \\
\text { DATA (IF USED) }\end{array}$ \\
\hline Optional & $\begin{array}{l}\text { (Section 3) } \\
\text { ECG LEAD DEFINITION }\end{array}$ \\
\hline Optional & $\begin{array}{l}\text { (Section 4) } \\
\text { QRS LOCATIONS (IF REFERENCE BEATS ARE } \\
\text { ENCODED) }\end{array}$ \\
\hline Optional & $\begin{array}{l}\text { (Section 5) } \\
\text { ENCODED REFERENCE BEAT DATA IF } \\
\text { REFERENCE BEATS ARE STORED }\end{array}$ \\
\hline Optional & $\begin{array}{l}(\text { Section } 6) \\
\text { "RESIDUAL SIGNAL" AFTER REFERENCE BEAT } \\
\text { SUBTRACTION IF REFERENCE BEATS ARE } \\
\text { STORED, OTHERWISE ENCODED RHYTHM DATA }\end{array}$ \\
\hline Optional & $\begin{array}{l}\text { (Section 7) } \\
\text { GLOBAL MEASUREMENTS }\end{array}$ \\
\hline Optional & $\begin{array}{l}\text { (Section 8) } \\
\text { TEXTUAL DIAGNOSIS FROM THE } \\
\text { "INTERPRETIVE" DEVICE }\end{array}$ \\
\hline Optional & $\begin{array}{c}\text { (Section 9) } \\
\text { MANUFACTURER SPECIFIC DIAGNOSTIC AND } \\
\text { OVERREADING DATA FROM THE } \\
\text { "INTERPRETIVE" DEVICE }\end{array}$ \\
\hline Optional & $\begin{array}{c}\text { (Section 10) } \\
\text { LEAD MEASUREMENT RESULTS }\end{array}$ \\
\hline Optional & $\begin{array}{l}\text { (Section 11) } \\
\text { UNIVERSAL STATEMENT CODES RESULTING } \\
\text { FROM THE INTERPRETATION }\end{array}$ \\
\hline
\end{tabular}

The SCP standard was implemented by a couple of European and American Manufacturers. Practical experience during implementation and in the field 
revealed both: it's usability, e.g. for telemetric applications as well as for data volume effective storage and retrieval ( e.g. in the OEDIPE project [3]).

There are three format options for ECG data within the SCP standard:

- Storage of "Original” ECG data

- Redundancy Reduction of ECG data

- SCP "High" compression. The principle of this compression method has been described in Annex C of [1]

\section{Methodology of the Content and Format Checker}

Comparison of SCP records from different devices of different manufacturers revealed differences in the implementations which made these records not really interoperable. To identify which of the records resembles with the SCP specifications compliance testing is necessary. Essentially the compliance tests has to cover the following tests:

- The content of the SCP record

- The format and structure of the SCP record

- The messaging mechanisms if records are communicated according to the SCP specifications.

Our work has focused on testing content and format of SCP records for compliance with the SCP specifications. Since this standard was developed in 1993 due to technological progress the previous messaging mechanisms have been changed and are not used in today's ECG devices.

There is an interdependence between content and format checking. Because of this interdependence an integrated testing is advisable. We developed software tools written in ANSI C for integrated content and format checking. There is a "Test-Driver" for file input/output operations, the analysing libraries and a common GUI. This system allows for both, testing SCP version 1.0 and the revised version 1.3. Since records to be tested may completely deviate from the SCP format throughout all test phases basic plausibility checks have been implemented to avoid crashes of the test functions.

\subsection{Testing the content of SCP records}

The purpose of the content test is to verify the content of all parts of a SCP file. The test starts with basic plausibility checks after reading the input data record:

- The program checks the compliance of record length and the length information.

- The program checks if the first section is the SCP pointer section (section Id. 0) using the section Id, the section length information and the SCP version number.

If one of these checks fails the program terminates with an error message/code. Otherwise the program continues and tabulates the version number of the tool, the name of the input file, the global CRC and the record size.

Subsequently the program provides the content of the SCP pointer section, e.g., section Id., length and index for each present section in a table. The second part of the content table depicts the content of the SCP section ID headers of all present sections (e.g. the section ID,. the length, the SCP version identification and the CRC for each section). Comparing the entries for length's and section identifications of the two tables allows already a visual plausibility check.

Then the program continues to analyse the content of the other sections detected. The content of these sections depends very much on the format of each specific section. For each section a plausibility check on pointer and length information is to be performed.

The information in the output text file is used for verification and validation of SCP Compression/ Decompression and Formatting software.

\subsection{Testing the format of SCP records}

The aim of the format testing is to check in detail if the SCP format specifications are met. The format checker tool analyses in detail all items of each section for compliance. The program performs the following types of tests:

a) Global Tests:

- Check Length of the record

- Check Global CRC

- Mandatory SCP sections 0 and 1 present

- The first section must be section 0

If more than two of these checks fails the program terminates with an error message/code, because the record is not compliant with the SCP specification.

b) Common Tests for all present SCP sections:

- Check that the pointer is located within the SCP record

- Check that the pointer + section length is located within the SCP record

- Check section ID between entry in section 0 and the section header

- Check section length between entry in section 0 and the section header

- Check for even section length

- Check for the SCP section version number (only for section id 0..11)

- Check for the SCP section protocol version number (only for SCP version > 1.0)

- Check for the local CRC-CCITT 
c) Section dependent Tests (examples)

c.1) Section dependent Format Tests for SCP Section 0 Plausibility of SCP section Ids, length's and indexes are checked.

c.2) Section dependent Format Tests for SCP Section 1

These tag-oriented tests have been implemented to verify content and to compare the format with the SCP specifications of up to 37 different tags.

c.3) Section dependent format tests for SCP Section 2 The program checks that if the number of Huffman tables $\neq 19999$, all tables and all code structures are defined, for each structure: number of bits in entire code is $\geq$ number of bits in prefix and if there is a table mode switch to an undefined Huffman table.

c.4) Section dependent Format Tests for SCP Section 3

The program checks, if the lead definitions are correct.

c.5) Section dependent Format Tests for SCP Section 4

Plausibility of the protected QRS areas and the subtraction intervals are tested by the program.

c.6) Section dependent Format Tests for SCP Section 5 and SCP section 6

Encoding parameters for the reference beat and the ECG data are checked for compliance with the specifications.

\section{Results}

The results of the SCP content checker are depicted as an example in Figures 1 and 2:

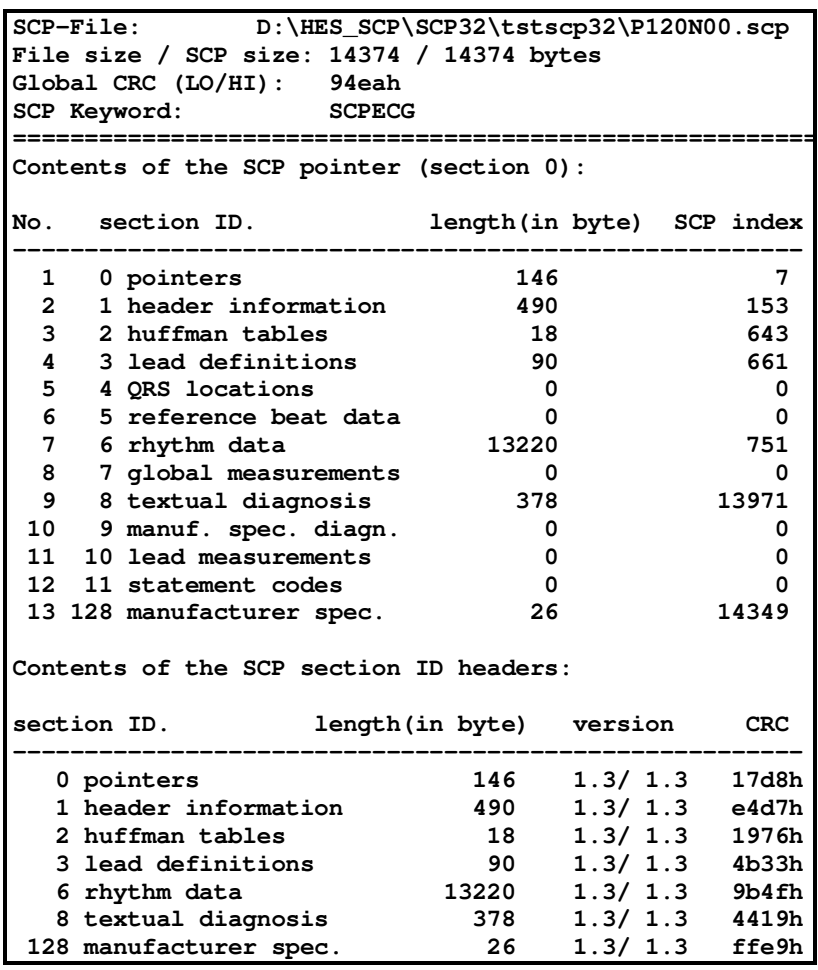

Figure 1. Example of the content of the SCP pointer section 0 and the SCP section headers.
The upper part of Figure 1 contains file name, record size, global CRC and SCP ID. The middle part depicts the sections entered into section 0 as specified according to the SCP standard. This part gives their size and represents a "table of contents" of the SCP file. The lower part displays the header information of each filled section.

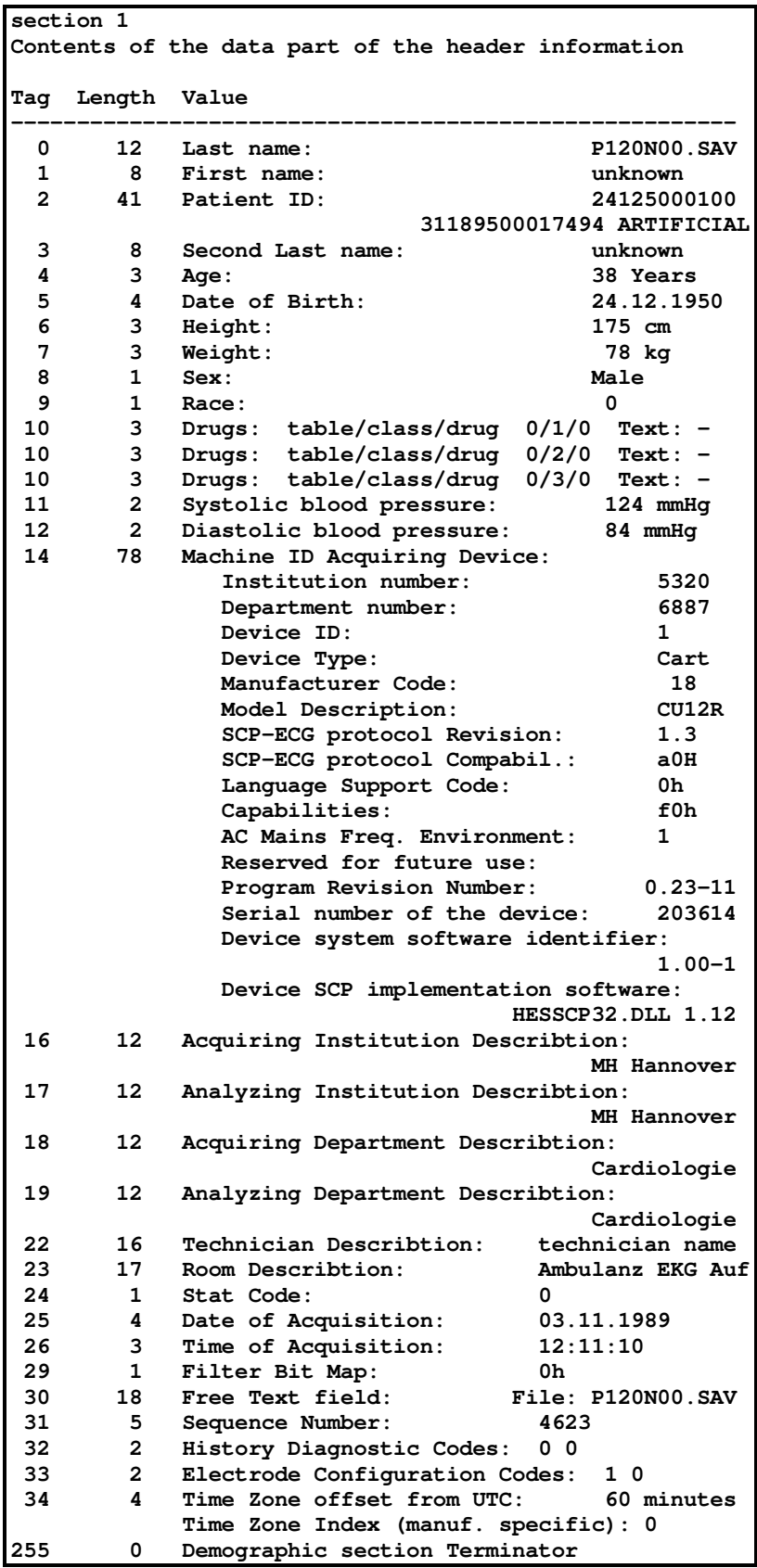

Figure 2. Example of the content of the SCP header information-Patient data/ECG acquisition data section 1.

As can be seen this section contains demographic patient data, information on drug classes, and there is 
space for storing/transfer blood pressure measurement. Furthermore there is space for detail device and data acquisition identification, information on the acquiring institution, the recording technician, time stamping and information on the patient history diagnostic code.

The results of the SCP format checker are depicted as examples in Figure 3 and 4. The text output file contains:

- Title and version of the format checking program

- Name of the checked SCP file

- Detected Errors and/or Warnings.

- Total number of format tests

- Number of detected format errors/warnings

The large number (in some cases more than 400) of performed tests is caused by check procedures, which need to be executed many times, e.g., test c.5 will be performed for each detected QRS complex and text field checks (within tests c.2) are provided for a large number (up to 17 ) of different text tags. Please note section 1 may contain 37 different tags. The number of tests may increase respectively.

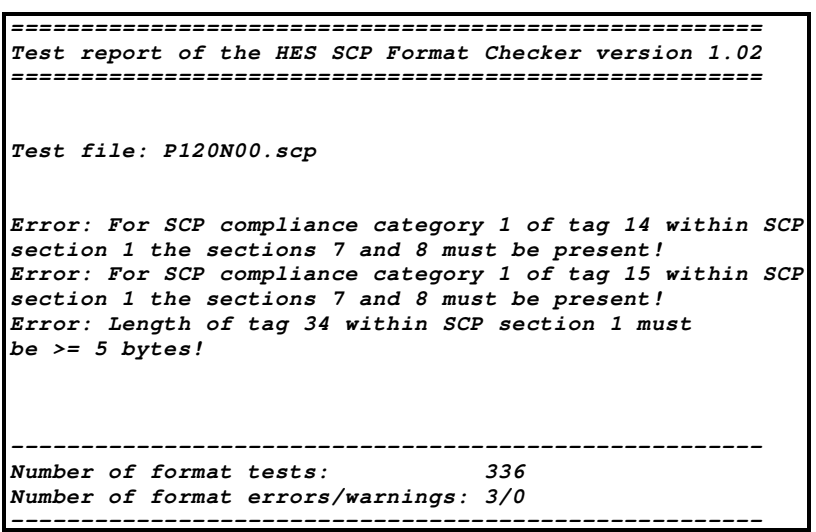

Figure 3. Example of the result of a Format checking test with errors.

336 tests have been performed. In this case, according to the entries for SCP compliance level within tag 14 and 15 section 7 (Global measurements) and section 8 (Textual diagnosis from the interpretative device) should be present, but they are not.

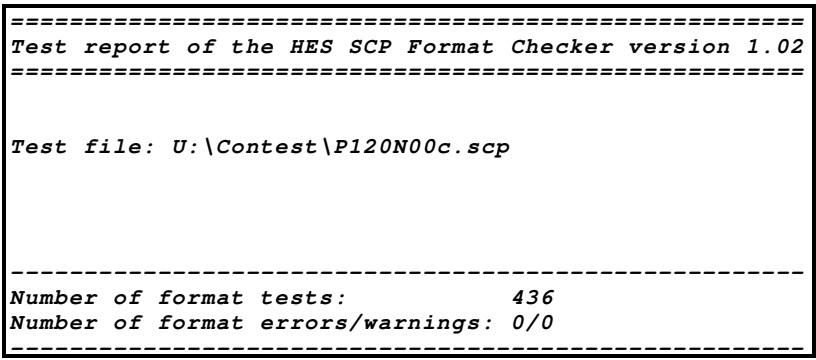

Figure 4. Example of the result of a Format checking test without errors/warnings.
Due to the different record content 436 tests were (automatically) performed, and no errors or warnings were detected. Warnings are given if, e.g., an empty text tag is present in section 1 , which is permitted within the standard but which makes no sense.

\section{Discussion and conclusions}

The presented tools are important for development as well as compliance testing of SCP formatted ECG records. They are necessary to promote the interoperability of important non-invasively gathered cardiac function information. This information is increasingly to be integrated into the electronic patient health record and to be interchanged between the various health care providers.

These software tools have been also provided for use within the European OpenECG project [4]. There already a significant number of compliance tests could be supported. For more information about the implementation of SCP see [5-6].

\section{Acknowledgements}

Part of this work has been carried out during the EU supported project OpenECG IST-2001-37711.

\section{References}

[1] Standard Communications Protocol for ComputerAssisted Electrocardiography. CEN European PreStandard ENV 1064, 1993.

[2] Willems JL, et al. Development of a Standard Communication Protocol for Computerised Electrocardiography. In: Duisterhout JS, Hasman \& R. Telematics in Medicine. North Holland: Elsevier Publishers B.V., IMIA, 1991:299-311.

[3] OEDIPE - AIM 2026, Open European Data Interchange and Processing for Computerised Cardiography, Demonstrator for Open Host to Host ECG Data Interchange Public Report, 1993-08-19, Deliverable 7

[4] OpenECG portal: http//www.openecg.net/

[5] R. Fischer, Chr. Zywietz How to Implement SCP: http://www.openecg.net/SCP_howto.pl

[6] R. Fischer, F. Chiarugi, Chr. Zywietz How to Implement SCP Part 2: http://www.openecg.net/SCP_howto_II.pl

Address for correspondence

Christoph Zywietz

BIOSIGNA Institute

Feodor-Lynen-Str.21 (Medical Park)

30625 Hannover/Germany

Zywietz.Christoph@Biosigna.de 